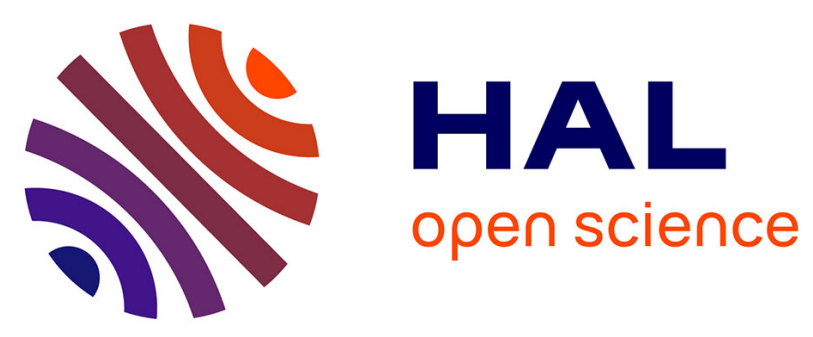

\title{
Plastic zone evolution during fatigue crack growth: Digital image correlation coupled with finite elements method
}

Jérôme Hosdez, Mederic Langlois, J-F Witz, N. Limodin, D. Najjar, E. Charkaluk, P. Osmond, A. Forre, F. Szmytka

\section{To cite this version:}

Jérôme Hosdez, Mederic Langlois, J-F Witz, N. Limodin, D. Najjar, et al.. Plastic zone evolution during fatigue crack growth: Digital image correlation coupled with finite elements method. International Journal of Solids and Structures, 2019, 171, pp.92-102. 10.1016/j.ijsolstr.2019.04.032 . hal-02309146

\section{HAL Id: hal-02309146 \\ https://hal.science/hal-02309146}

Submitted on 9 Oct 2019

HAL is a multi-disciplinary open access archive for the deposit and dissemination of scientific research documents, whether they are published or not. The documents may come from teaching and research institutions in France or abroad, or from public or private research centers.
L'archive ouverte pluridisciplinaire HAL, est destinée au dépôt et à la diffusion de documents scientifiques de niveau recherche, publiés ou non, émanant des établissements d'enseignement et de recherche français ou étrangers, des laboratoires publics ou privés. 


\title{
Plastic zone evolution during fatigue crack growth: Digital Image Correlation coupled with Finite Elements Method
}

\author{
J. Hosdez ${ }^{\mathrm{a}}$, M. Langlois ${ }^{\mathrm{a}}$, J-F. Witz ${ }^{\mathrm{a}}$, N. Limodin ${ }^{\mathrm{a}}$, D. Najjar ${ }^{\mathrm{a}}$, \\ E. Charkaluk ${ }^{\mathrm{b}}$, P. Osmond ${ }^{\mathrm{c}}$, A. Forre ${ }^{\mathrm{c}}$, F. Szmytka ${ }^{\mathrm{d}}$ \\ ${ }^{a}$ Univ. Lille, CNRS, Centrale Lille, Laboratoire de Mecanique, Multiphysique et \\ Multiechelle (LaMcube) FRE 2016, 59000 Lille, France. \\ ${ }^{b}$ Laboratoire de Mecanique des Solides (LMS), UMR CNRS 7649, 91762 Palaiseau, France. \\ ${ }^{c}$ PSA Group, Direction de la Recherche et de l'Innovation Automobile, Route de Gisy, \\ 78943 Velizy-Villacoublay Cedex, France. \\ ${ }^{d}$ Institut des Sciences de la Mecanique et Applications Industrielles (IMSIA), UMR \\ EDF/CNRS/CEA/ENSTA 9216, 91762 Palaiseau, France.
}

\begin{abstract}
Nonlinearities effects at the crack tip, due to the elastic-plastic material behavior, impact the crack growth rate and path. This paper is devoted to the study of the plastic zone evolution in the crack tip region. The methodology relies on coupling an elastic-plastic Finite Elements Method (FEM) model and experimental displacements measured by Digital Image Correlation (DIC). These latter are introduced as Dirichlet boundary conditions in the finite elements analysis. The considered FEM domain is constant, i.e. the same mesh with a centered crack is moved to each new crack tip position deduced from DIC. The new boundary conditions are updated and the residual stresses and plastic strains of the former computation are interpolated and actualized on the mesh shifted to the new crack tip position in order to incorporate them in the numerical model. The coupling method allowed applying experimental boundary conditions in order to be as close as possible to real experimental conditions and to observe the plasticity evolution from small to large scale yielding conditions. A fatigue test was conducted to validate the proposed approach. The identification residues are proved to be lower than those obtained with an experimental displacements projection onto Williams' series basis, which is a method commonly used with DIC. The coupling results present an attractive similarity
\end{abstract}


with Irwin's model regardless of the crack length. Thus, the definition of the mask needed for the displacements fields projection on Williams' model can be deduced with a reliable estimate of Irwin's plastic radius.

Keywords: Non-linear Fracture Mechanics, Plasticity, Fatigue, Crack propagation, Digital Image Correlation, Finite Elements Method, Ductile Cast Iron

\section{Introduction}

Fatigue crack propagation is often associated with small scale yielding conditions. In this case, the plastic zone is constrained by the elastic bulk. However for the larger scale yielding situations, plasticity can no longer be neglected. Indeed it induces a competition between the intrinsic damage mechanisms occurring ahead of the crack front and the extrinsic ones provoking a crack shielding. Furthermore, the influence of this latter effect increases when the material is submitted to overloads, leading to larger compressive residual stresses in front of the crack tip. History effects involve cyclic plasticity at the crack tip and as a result it should be considerably impacted by the material constitutive law [3:3]. Therefore, having a reliable method to estimate the plasticity at the crack tip would improve crack growth predictions. Nonetheless, the plasticity identification at the crack tip is relatively complex whether from numerical or experimental ways.

From the numerical point of view, the Finite Elements Method (FEM) has been applied successfully in a myriad of structural problems; however, it has shown some limits in modelling moving interfaces such as cracks. In the two last decades, various numerical methods were proposed to deal appropriately with these moving interfaces in fracture mechanics especially the eXtended Finite Elements Method (X-FEM) approach [4, 26]. The X-FEM employs the partition of unity in two ways: first the displacement jump across the crack is considered, and then the approximation is enriched by considering the asymp- 
totic fields close to the crack tip.

It was applied to many problems in the framework of Linear Elastic Fracture Mechanics (LEFM) such as elastic fatigue crack growth [26], by considering in addition the friction between the crack lips [I4], 3D fatigue crack growth with level set approach [27] and dynamic crack propagation [5, 37]. More recently, the X-FEM has been extended to deal with plasticity [15].

Nonetheless, the computation boundary conditions could be problematic because they are not always perfectly known. In this case, reliable results on the fatigue crack growth or plastic zone shape could not be obtained. To circumvent this difficulty, an hybrid approach coupling experimental information and numerical simulations to determine plastic zone propagation during fatigue crack growth is proposed.

Different techniques, in particular the full-fields measurements, were employed to investigate the fatigue crack propagation and the plastically deformed region surrounding the crack. During the last decades, the use of kinematic measurements coupled with micro-tomography for 3D cases and optical observation for 2D cases via Digital Volume Correlation/Digital Image Correlation (DVC/DIC) has been developed considerably [3, [7, ㅍ, 38, 43]. Lachambre et al. [2:3] used X-ray micro-tomography coupled with Digital Volume Correlation to highlight the plastic zone in ductile cast iron. The observation was achieved with DVC computations between the 3D images taken at the minimum load of initial state and at the minimum load at different stages of fatigue crack growth. Three-dimensional information would be relevant to apprehend the shape of the crack front or the plasticity conditions both in the bulk and on the surface of the fatigue specimen. Nonetheless, some limits could be mentioned such as the necessity to use specimens with small dimensions, i.e. with a cross-section of only a few millimeters, in order to ensure a sufficient tomography resolution. Even with laminography where the condition on the width of flat specimens could be alleviated, the thickness remains limited to about $1 \mathrm{~mm}$ when a high resolution of the microstructural heterogeneity is looked for [41]. At this scale, 
significant local heterogeneities can be observed [10] in particular in ductile cast irons where the grain size is around 80 microns [30] and they do influence the growth of the relatively short cracks. The understanding of phenomena due to plasticity can thus become more complex, that is why a two-dimensional analysis at a coarser scale and on larger specimens is preferred in a first step to the use of micro-tomography.

In two dimensions, DIC is one of the most used technique and several strategies can be retained to bring forward plasticity. A direct way consists in displacements fields' derivation and the use of a specific criterion [44, 45]. Nevertheless, strain fields could contain some noise that will impact the plastic zone determination. Indirect ways can be preferred by using an appropriate model as a projection basis, the Williams' expansion series for example [46] . Confined plasticity cases can be dealt with [1, $18,[28]$ but it is less suitable for large scale yielding conditions [20, 25]. An alternative methodology to describe the local elastic-plastic effects was proposed by Decreuse et al. [[2] which consists in decomposing DIC displacements into an elastic part and a complementary one. The decomposition was conducted with a Proper Orthogonal Decomposition (POD) method, commonly used in the analysis of turbulent flows [6]. Finally, the measured displacements fields can also be imposed as Dirichlet boundary conditions to an elastic-plastic FEM model. It requires that the material constitutive law is known and especially the kinematic hardening that can play a major role in the cyclic behavior. This methodology has been used by Roux-Langlois et al. [39] without taking into account the loading history during the crack propagation. Thus, the DIC-FEM formulation constitutes a good tool to extract linear and non-linear features in the crack tip vicinity in a two-dimensional case.

The main objective of this article is to propose a method to quantify plastic domains at the crack tip. Results are compared with Irwin's model and the question of relevance of the use of Irwin's plastic radius for integration 
mask definition in projective approach is investigated. The method is based on a coupling between experimental displacements fields and elastic-plastic computations, provided by digital images correlation and finite elements methods respectively. This hybrid approach is applied to a fatigue test conducted on a Single Edge Notch Tension (SENT) specimen of ductile cast iron. The test results are analyzed with both Williams' series projection and the proposed methodology. In the following sections, the material is first described as well as the experimental set-up and the full-fields projection on Williams' basis (Section 2). The DIC-FEM coupling method principle is discussed in Section 3. Section 4 presents the application of the strategy to the above mentioned fatigue test and the final section gives some conclusions and possible future works.

\section{Material and test conditions}

\subsection{Material and fatigue specimen}

The studied material is a Spheroidal Graphite cast Iron (SGI) denoted as SiMo, enriched in silicon and molybdenum, commonly used in the automotive industry for exhaust manifolds manufacturing. The heterogeneous microstructure presents a ferritic matrix with graphite nodules that are randomly distributed with a mean diameter typically close to 25 micrometers (Fig. $\mathbb{1}$ ).

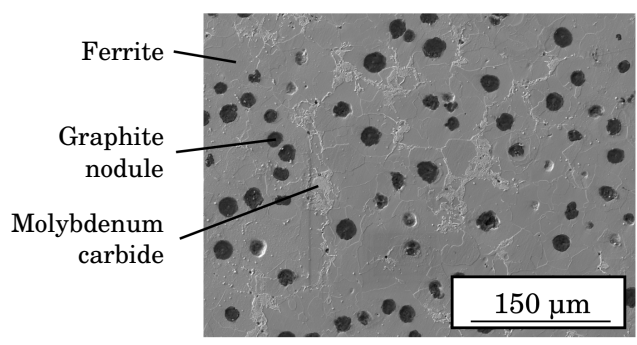

Figure 1: Spheroidal graphite cast iron observed with scanning electron microscopy.

The cast iron chemical composition is reported in Table m. The material was provided in the form of foundry bars, obtained by gravity die casting, with a length of $200 \mathrm{~mm}$ and a diameter of $25 \mathrm{~mm}$. A Single Edge Notch Tension 
(SENT) specimen, with a thickness of $3.8 \mathrm{~mm}$, a length of $140 \mathrm{~mm}$ and a width of $18 \mathrm{~mm}$ was cut from the cast bars (Fig. च). A straight-through notch with a total length of $3.4 \mathrm{~mm}$, an opening angle of $60^{\circ}$ at the notch tip and a height of $1 \mathrm{~mm}$ was cut by electro discharge machining.

\begin{tabular}{cccccc} 
& C & Si & Mo & Mn & P \\
\hline SGI & 3.4 & 3.89 & 0.57 & 0.15 & 0.025 \\
\hline
\end{tabular}

Table 1: Chemical composition of the studied ductile cast iron (weight \%).

\subsection{Test conditions}

The SENT specimen was tested under pure mode I loading until failure. Before conducting the fatigue test, a pre-cracking step was achieved with a load shedding procedure [16]. The process consists in decreasing the load to restrict the Stress Intensity Factor (SIF) increase during the fatigue crack propagation. The load shedding process is based on the Direct Current Potential Drop (DCPD) method. In doing so, a constant intensity current is applied through the specimen and the electrical potential across the crack is followed with a voltmeter at the maximum load of each cycle (Fig. 지).

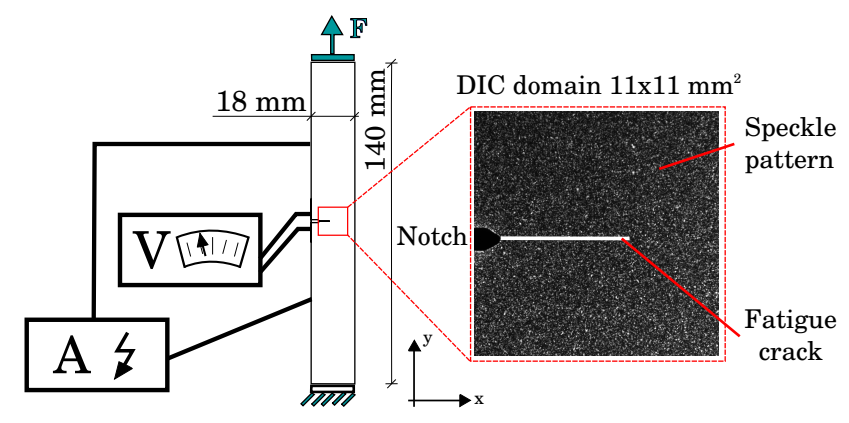

Figure 2: The studied zone of the SENT specimen with inputs/outputs for DCPD method and the speckle pattern for DIC.

When the crack extends, the electrical resistance rises as well as the measured potential. The relation between electrical potential and crack length has 
to be established. Then, the analytical relationship between the specimen geometry, i.e the crack length, and the SIF has to be obtained in order to monitor the SIF increase during crack propagation. In this work, a FEM calibration is chosen to estimate the crack length from electrical potential, and an analytical formula for SENT specimen [36] is used to compute the stress intensity factor. More details about the calibration steps can be found in [20]].

An AGILENT 344201 nano-voltmeter, which presents a high sensitivity of $100 \mathrm{pV}$, was used for the DCPD technique. The specimen is electrically insulated from the testing machine by positioning elastomeric plates, between the grips and the SENT specimen.

The test piece is observed with a camera triggered to the load cell of the fatigue machine. Pictures, with a definition of 2048 by 2048 pixels, are taken every 50 cycles at the maximum and the minimum load values. A telecentric lens is employed to avoid false perspectives, distortions and to reach a pixel size of 5.25 $\mu \mathrm{m}$ (Fig. $\mathbb{Z}$ ). The images were analyzed to determine the displacement fields by using Digital Image Correlation (DIC) technique. The present approach is local, based on a non-continuous description of the displacement field. More precisely, each images couple are correlated by an Optical Flow method with Integrated kinematics within independent Blocks (OFIB). A linear system, computed for a specific transformation, is solved by block regardless of the neighboring ones. Consequently, this methodology allows identifying heterogeneous displacement fields keeping basic kinematics, namely rigid body motions, at the block scale. The whole procedure is performed using YaDICs home-made programming platform and more details can be found in [40]. The DIC procedure can be achieved with $4 \times 4$ pixels windows ensuring a good spatial resolution (Fig. [3) thanks to the paint speckle pattern that was sprayed by air-brush before the fatigue test. 


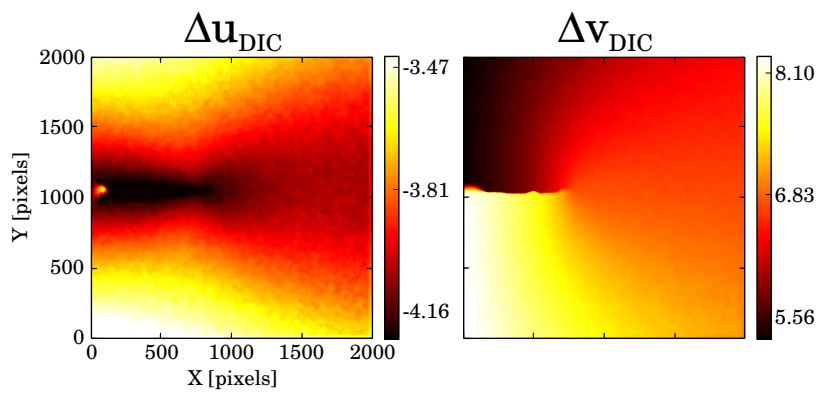

Figure 3: On the left, the horizontal displacements measured with DIC and on the right the vertical displacements $(1 \mathrm{pixel}=5.25 \mu \mathrm{m})$.

A one millimeter long sharp pre-crack was achieved during 200,000 cycles at constant maximum stress intensity factor $K_{\operatorname{Imax}}$ of $12 \mathrm{MPa} \sqrt{\mathrm{m}}$ with a frequency of $2 \mathrm{~Hz}$ and a load ratio of 0.1 . Then, during the propagation step, a sinusoidal loading profile is applied with a constant maximum load value of 6.8 $\mathrm{kN}$. The stress intensity factors range from the minimum to maximum crack length increases from 10 to $60 \mathrm{MPa} \sqrt{\mathrm{m}}$. The frequency and the load ratio are kept respectively at $2 \mathrm{~Hz}$ and 0.1 . Finally, the specimen failed around 80,000 cycles after the end of the pre-cracking step, with a final crack length close to $12 \mathrm{~mm}$.

\subsection{Projective approach}

In a previous article [20], an appropriate DIC displacements projection was conducted on Williams' basis. The limitations of the method, proving the necessity of DIC-FEM coupling, will be shown after presenting its main guidelines.

\subsubsection{General principles}

The projection methodology allows to extract linear and non-linear fatigue fracture features, especially the Stress Intensity Factor (SIF), in a homogeneous, isotropic and two-dimensional medium. The approach refers to the Williams' model which expresses displacements in a cracked medium, submitted to a mixed mode cracking in the case of confined plasticity [18], in the form of expansion series [46]: 


$$
\begin{aligned}
& u=\sum_{-\infty}^{\infty} \frac{r^{n / 2}}{2 G} \omega_{I}^{n}\left[\left(\kappa+\frac{n}{2}+(-1)^{n}\right) \cos \frac{n \theta}{2}-\frac{n}{2} \cos \frac{(n-4) \theta}{2}\right]+ \\
& \sum_{-\infty}^{\infty} \frac{r^{n / 2}}{2 G} \omega_{I I}^{n}\left[-\left(\kappa+\frac{n}{2}-(-1)^{n}\right) \sin \frac{n \theta}{2}+\frac{n}{2} \sin \frac{(n-4) \theta}{2}\right] \\
& v=\sum_{-\infty}^{\infty} \frac{r^{n / 2}}{2 G} \omega_{I}^{n}\left[\left(\kappa-\frac{n}{2}-(-1)^{n}\right) \sin \frac{n \theta}{2}+\frac{n}{2} \sin \frac{(n-4) \theta}{2}\right]+ \\
& \sum_{-\infty}^{\infty} \frac{r^{n / 2}}{2 G} \omega_{I I}^{n}\left[\left(\kappa-\frac{n}{2}+(-1)^{n}\right) \cos \frac{n \theta}{2}+\frac{n}{2} \cos \frac{(n-4) \theta}{2}\right]
\end{aligned}
$$

with $\omega_{I}^{n}$ and $\omega_{I I}^{n}$ Williams' coefficients for the $n^{\text {th }}$ order in mode I and mode II respectively, $(r, \theta)$ the polar coordinates, $G$ the shear modulus and $\kappa$ the Kolossov constant corresponding to $\frac{(3-\nu)}{(1+\nu)}$ for plane stress and $3-4 \nu$ for plane strain cases.

Depending on the $n^{\text {th }}$ order, the field presents a specific significance. Indeed the first order corresponds to the analytical solution of Westergaard so that it enables to identify the Stress Intensity Factor: $K_{I}$ for the opening mode and $K_{I I}$ for the in-plane shear mode. The projection method proposed in a previous work [20] can be summarized as follows:

1. The crack length is computed by thresholding the crack discontinuity via the displacements gradient measured with DIC in the loading direction.

2. As the crack tip location is obtained, the analytical expression of Williams can be generated (Eq. $\square$ and 『).

3. The SIFs ranges, $\Delta K_{I}$ and $\Delta K_{I I}$, are estimated by the projection of experimental displacements on Williams' basis. Finally, this step corresponds to a minimization of the difference between experimental and analytical fields via a least squares method. 


\subsubsection{Results and limits in terms of plasticity}

The projection procedure based on Digital Image Correlation was undertaken with:

- $4 \times 4$ and $8 \times 8$ pixels DIC window sizes for the crack length and SIF identifications respectively. The uncertainties for the mentioned image correlation window sizes, corresponding to the standard deviation of displacements, are 0.013 and 0.011 respectively.

- A range of Williams' orders from 1 to 7 to investigate the last stages of crack propagation where boundary conditions are predominant.

- An identification zone to restrict the plasticity and boundary conditions impacts with an inner radius set at 150 pixels and an external one of 800 pixels. A sensitivity study was carried out for the external radius and the inner one was set from Irwin's plastic radius cited in $[8]$.

The obtained values for the crack tip location and for the SIF allow identifying the fatigue crack propagation law. Indeed, the crack growth rate $d a / d N$ can be expressed as being proportional to the SIF range [32] using the well known Paris' law:

$$
\frac{d a}{d N}=C \Delta K^{m}
$$

The projective approach provides Paris' coefficients $C$ and $m$ close to those of the DCPD and of the literature for a ductile cast iron at load ratio $R$ of 0.1 (Tab. 『).

\begin{tabular}{|c|c|c|}
\hline & $C$ & $m$ \\
\hline Projective DIC [20] & $10^{-8.9}$ & 4.03 \\
\hline DCPD $[20]$ & $10^{-8.87}$ & 4.07 \\
\hline Dierickx [1]3] & $10^{-9.0}$ & 3.81 \\
\hline
\end{tabular}

Table 2: Identified parameters of Paris' law and comparison with literature values [[13]. 
Nonetheless, the Stress Intensity Factor formulation is defined assuming confined plasticity, near the crack tip while the plasticity may increase significantly during crack propagation of a standardized testing, i.e. with a constant maximum loading. This raises the question of what is the impact of plasticity on a particular metrological medium. The DCPD method has a more elastic intrinsic behaviour than image correlation, because it is based on a single analytical law assuming a perfectly elastic medium. The projective DIC presents a certain richness of information through the fields obtained and therefore enables to mask the displacement values impacted by the plasticity at the crack tip. Thus, the influence of the latter on identifications may be reduced.

Furthermore, DIC enables to investigate this non-linear effect during the fatigue test. The difference between experimental (raw DIC measurement) and analytical (projection onto Williams' basis) displacements fields, which is called residue hereafter, gives some information on plasticity. Indeed a residue can occur because plasticity is not taken into account in the projection basis, i.e. in Williams' expansion series. During the experiment, the residue increased gradually close to the crack tip (Fig. $⿴$ ) revealing what we assume to be a part of the local cyclic plasticity. At last steps of the crack propagation, with a crack length of $10.9 \mathrm{~mm}$, a large residue is observed within a millimetric zone and its contribution to the crack growth rate may not be negligible anymore.
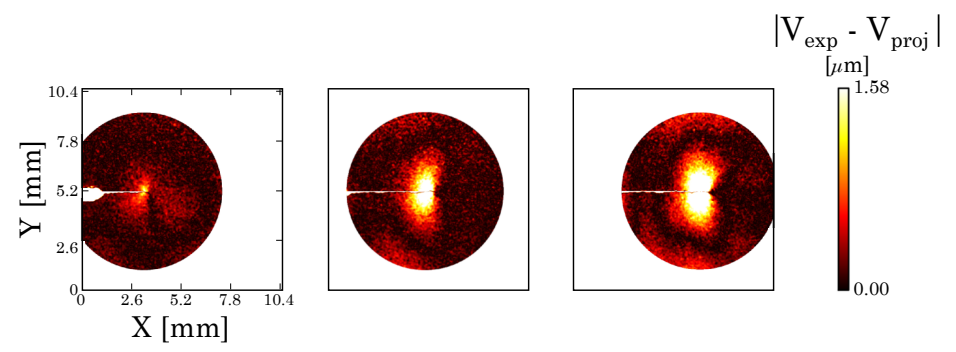

Figure 4: The vertical residue for crack lengths of $6.1 \mathrm{~mm}$ (corresponding to 55,000 cycles), $8.9 \mathrm{~mm}(74,200$ cycles $)$ and $10.9 \mathrm{~mm}(76,500$ cycles $)$. The window size is close to $10 \times 10 \mathrm{~mm}^{2}$. 
Irwin's model gives a relationship between the monotonic plastic zone radius $R_{p}$, the stress intensity factor $K_{I}$ and the material yield strength $\sigma_{y}$ :

$$
R_{p}=\frac{1}{2 \pi}\left(\frac{K_{I}}{\sigma_{y}}\right)^{2}
$$

If we compare Irwin's model with the residue localization, we can observe a good match [20] highlighting the fact that plasticity certainly contributes for an important part of the residue rise. Nonetheless residues may indicate other issues especially some non-considered 3D effects such as out-of-plane aspects or a non-straight crack propagation. Stereo-DIC, which allows a 3D displacements measurement on the surface [3I], was tested during a fatigue test, but no significant out-of-plane displacements were observed. Moreover, micro-tomography was also employed to reveal the crack shape. In some specimens, a curved crack front was noticed with a difference between the core and the surface of almost $1 \mathrm{~mm}$ in crack length. Such a deviation, not considered in the Williams model, should also contribute to the residue values obtained.

Despite the good correspondence between residues localization and Irwin's model, the projective approach seems not sufficient to qualify the fatigue crack growth for the highest SIF values. Indeed in elastic-plastic fracture mechanics, the asymptotic fields are not analytically known. To overcome this limit, a coupling between experimental information, provided by DIC, and FEM computations is explored in the present work to go further in the crack growth study.

\section{DIC-FEM coupled approach}

In the following, a hybrid method based on DIC-FEM coupling is developed to assess the crack tip plasticity during crack growth and is employed to analyze the previously described experiment. First, the general principles are discussed and then the finite elements model is outlined. 


\subsection{General principles}

The main idea is to propose a solution to characterize plasticity by taking into account the experimental information from DIC results in FEM computations.

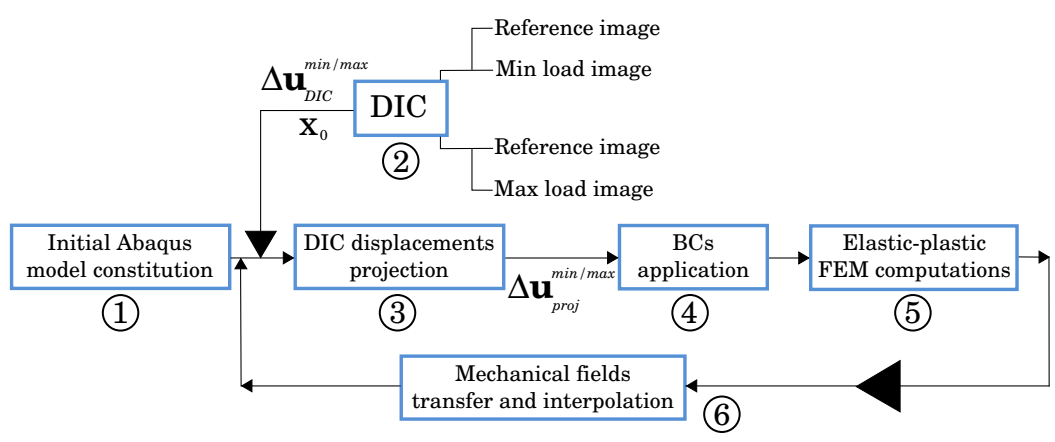

Figure 5: Main steps of the DIC-FEM strategy.

The details of the suggested methodology can be summarized as follows (Fig. 回):

1. The first step consists in defining the FEM elastic-plastic model with its specific material parameters and the geometry with its associated mesh. The particular characteristics are that the crack tip is centred on the FEM model and the mesh is kept constant, i.e. no update is considered (Fig. [6). This approach is feasible only if the crack propagates with no bifurcation and nor twisting.

2. Then, Digital Image Correlation is used to provide the boundary conditions every 50 cycles. DIC is carried out in two steps for each considered fatigue cycle: at the loading where $\Delta \mathbf{u}_{D I C}^{\max }$ is computed between the reference image, i.e. without loading, and the picture at the maximum load and at the unloading step where $\Delta \mathbf{u}_{D I C}^{\min }$ is computed between reference image and that at the minimum load.

3. Experimental displacements fields contain some noise which could perturb FEM computations. Therefore, the projected displacements, obtained with the sought Williams' parameters (Section 2.3), are retained 


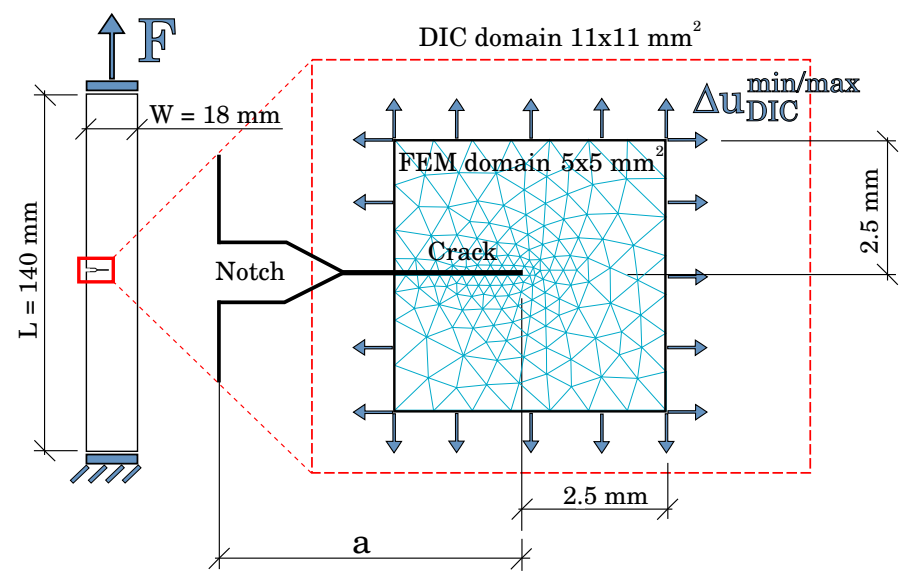

Figure 6: Geometry of the SENT specimen and boundary conditions with the DIC and FEM domains represented in dotted line and solid line respectively.

as boundary conditions in calculations. Besides, the use of a mask allows constraining the plasticity impact in the projection as the Williams' model assumes an elastic medium. Thus, the projected displacement fields, which can be assumed elastic especially far from the crack tip (Fig. 団), can be used as Boundary Conditions (BC) on the model perimeter to reduce the noise as compared to the use of raw displacement fields from DIC.

4. Next, the projected displacements at the loading $\Delta \mathbf{u}_{\text {proj }}^{\max }$ and unloading $\Delta \mathbf{u}_{\text {proj }}^{\min }$ steps are interpolated on the boundary nodes of the FEM domain and are used as Dirichlet BC in FEM computations.

5. The elastic-plastic computation is run with Abaqus. The FEM model is described in Section 3.2.

6. Afterwards, the considered FEM domain is moved to the new crack tip position, obtained from image correlation, and the new boundary conditions are updated with Williams' fields of the corresponding instant. Thus, the mesh procedure is efficient with one generation at the beginning of the DIC-FEM coupled approach and without modification during crack propagation. The whole updating strategy is able to deal with the evolving non-homogeneous loading conditions of the fatigue test. 
This kind of identification process has already been employed and its efficiency was highlighted by Roux-Langlois et al. [39]. Nevertheless in this latter case, history effects were not taken into account during the crack propagation. Herein, at the end of each calculation, the residual stresses and plastic strains are calculated by FEM as internal variables. After a crack propagation event, these tensors are transferred to the new mesh and treated as initial fields in the next DIC-FEM coupling computation (Fig. [).

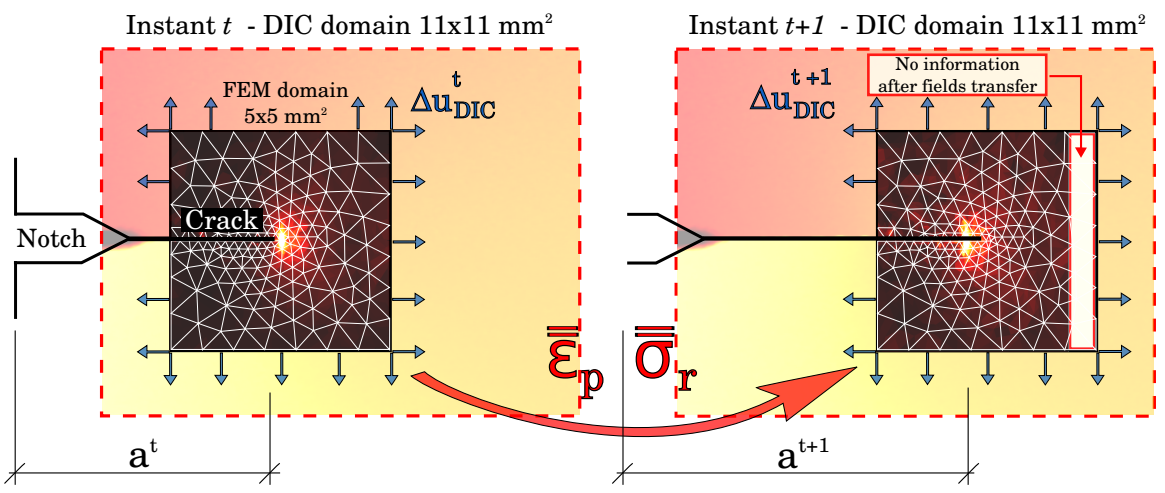

Figure 7: The transfer and interpolation procedures which carry the plastic strain and residual stresses from the old mesh to the next one at the new crack tip position.

Finally, the DIC-FEM coupling process is led with a minimum crack length increment of 10 pixels (corresponding approximatively to $50 \mu \mathrm{m}$ ) allowing to deal with a great number of cases within a reasonable time. This strategy was preferred to a temporal increment, i.e. in terms of cycles, as large scale yielding occurs in a few cycles at the end of the test. The procedure was fully automated using a Python script coupled with ABAQUS and requires a few minutes to treat one boundary conditions case. 


\subsection{FEM model description}

\subsubsection{Material parameters}

A simple elastic-plastic constitutive law for the crack tip region is preferred as the goal is to evaluate the process relevance in reasonable computation times. The Young's modulus and the yield strength are obtained from monotonic tensile tests. A linear kinematic hardening is retained for the elastic-plastic constitutive behavior. This latter leads to a plastic accommodation in one loading cycle. As a first step, the kinematic hardening parameter is considered equal to the tenth of the Young's modulus. Furthermore, the importance of the kinematic hardening will be discussed in Section 4. The material parameters used for the computations are reported in Table [1.

\begin{tabular}{cc} 
Young's modulus $E(\mathrm{GPa})$ & 180 \\
\hline Poisson's ratio $\nu$ & 0.28 \\
\hline Yield strength $\sigma_{y}(\mathrm{MPa})$ & 420 \\
\hline Kinematic hardening parameter $C(\mathrm{GPa})$ & 18 \\
\hline
\end{tabular}

Table 3: Parameters of the elastic-plastic constitutive behavior employed for FEM computations.

\subsubsection{FEM mesh}

The geometry and dimensions of the specimen are given in Figure [all the dimensions are in $\mathrm{mm}$ ) and those of the FEM mesh, are shown in Figure 8. The finite elements model was developed with the ABAQUS code. A plane stress condition is supposed due to the DIC use which enables only surface measurements. Thus, a 2D finite elements model is generated for the DIC-FEM coupling approach, corresponding to a square with an area of $5 \times 5 \mathrm{~mm}^{2}$. The mesh includes a centred crack perfectly horizontal, as the specimen is submitted to a mode I cracking conducting to limited crack bifurcation. Besides, DIC results acquired during the fatigue test confirmed the straight crack propagation (Fig. 3). 


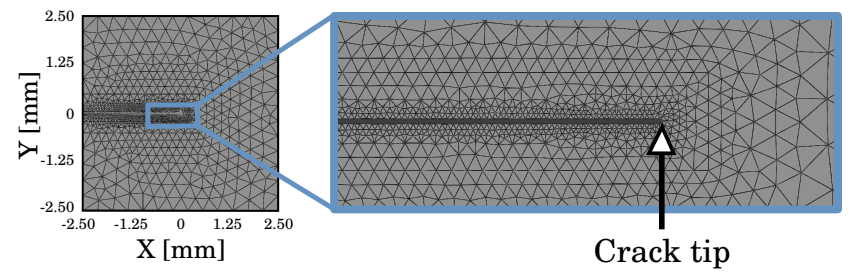

Figure 8: The whole model mesh $\left(5 \times 5 \mathrm{~mm}^{2}\right)$ with mesh refinement details around the crack $\operatorname{tip}\left(100 \mathrm{x} 40 \mu \mathrm{m}^{2}\right)$.

As the method takes into account plasticity at the crack tip, the plastic zone is supposed to propagate with the crack and it appears useful to have a fine knowledge of the stress state in the elements in the vicinity of the crack tip. Therefore, the mesh was greatly refined close to the crack tip with an element size around $1 \mu \mathrm{m}$. On the contrary, zones away from the crack tip are represented with less but enough elements to define accurately the boundary conditions provided by DIC. Although these are not the most suitable finite elements, linear triangular with three nodes are used for time consuming considerations. Nonetheless, the model includes approximately 7,500 triangles to accurately deal with plasticity at the crack tip. Solanki et al. [42] conducted a review of the study of crack closure, due to the plasticity, using the finite element method. According to his work, several types of elements can be used, including triangular elements. Solanki also specified that whatever the type of elements selected, their size must be a fraction of the size of the cyclic plastic zone. Finally, as the fatigue test is conducted at a load ratio of 0.1 , contacts of the crack lips are supposed to be limited and they are not taken into account in computations. Indeed, preliminary computations were run and no crack lips inter-penetrations were observed.

\section{Application to a fatigue test}

The DIC-FEM coupling approach is applied to the fatigue test case which was introduced in Section 2. In the following part, noise is highlighted during 
preliminary computations and the way to deal with it is discussed. Then, a comparison between the displacements fields' projection and the coupling approaches is shown. Next, the importance of the constitutive law is discussed. Finally, DIC-FEM coupling results are compared with those of Irwin's model (Eq. 四) in terms of plastic zone size.

As a reminder, the fatigue specimen is submitted to a mode I cyclic loading from a minimum load $F_{\min }=0.68 \mathrm{kN}$ to a maximum one $F_{\max }=6.8 \mathrm{kN}$. Figure $\mathbf{9}$ shows the measured crack length over cycles with DCPD and DIC. Results for both methods are in good agreement for all the test.

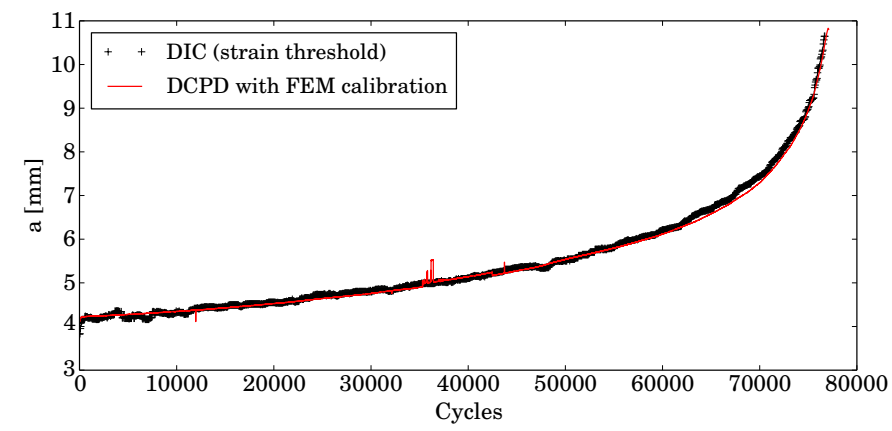

Figure 9: Measured crack length with DIC and DCPD over cycles.

The beginning and the end of the coupling approach are determined by the size of FEM and DIC domains. Indeed, boundary conditions, which are known only within the DIC domain, have to be well defined on the whole perimeter of the $5 \times 5 \mathrm{~mm}^{2}$ FEM model. Therefore, the procedure begins for a crack length of 5.8 approximately and ends at $10 \mathrm{~mm}$, corresponding respectively to 55,000 and 77,000 cycles. Due to the use of linear kinematic hardening, an immediate stabilized stress state around the crack tip is established. Thus, only two cycles are applied to the specimen for every updated boundary conditions. Then, a minimum crack growth of $\Delta a=10$ pixels, which corresponds to the length of a dozen of finite elements close to the crack tip, is considered. Finally, the whole procedure, i.e. the loading cycle application plus the growth step is repeated 
around 60 times for the retained crack growth increment.

\subsection{Noise impact}

Preliminary calculations with the first boundary conditions case, corresponding to a crack length of $5.8 \mathrm{~mm}$, have clearly shown the existence of a numerical noise: some regions are activated near the crack lips though they are free surfaces. Moreover, the successive loading cycles induce a noise amplification. The equivalent plastic strains at unloading are shown on Figure $\mathbb{}$ for the first boundary conditions for different numbers of cycles: 2, 5 and 15 .
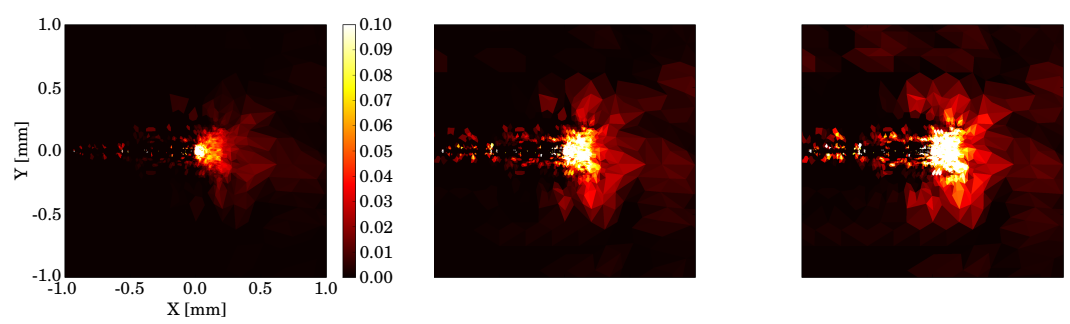

Figure 10: Magnifications of the equivalent plastic strain at unloading for $2^{\text {nd }}, 10^{t h}$ and $15^{\text {th }}$ cycles in the first boundary conditions case.

After transferring the stress and strain tensors from the old to the new mesh, the FEM model is not in mechanical equilibrium anymore, because the previous converged state was valid only for the old mesh. This phenomenon could potentially lead to significant errors. Indeed the stress and plastic strains tensors at the instant $t$ are saved and transferred to become 'inputs' (initial fields) for the next calculation at the instant $t+1$. However, mechanical equilibrium can be considered as established again using a small crack propagation increment, leading to limited errors induced by the fields transfer.

Herein, for each computation, only two cycles are considered because a Prager's linear kinematic hardening law [34] is used for the material behavior and conducts to an immediate stabilization. In this case, noise is limited, but a regularization step has to be added in the coupling procedure so that 
the required quantities, namely the residual stresses and the plastic strains, are filtered before introducing them in the next computation. As a regularization, corresponding to a mean or a median filter, could have a significant impact on the results, this possible influence will be assessed.

Due to the important irregularity of the FEM mesh (Fig. 8), a same number of integration points for the filtering step is considered. A while loop is employed with a radius that increases from each Gauss point to detect its neighborhood. Figure $\square$ highlights the results obtained for the equivalent plastic strains with different regularization methods after unloading for the first calculation.
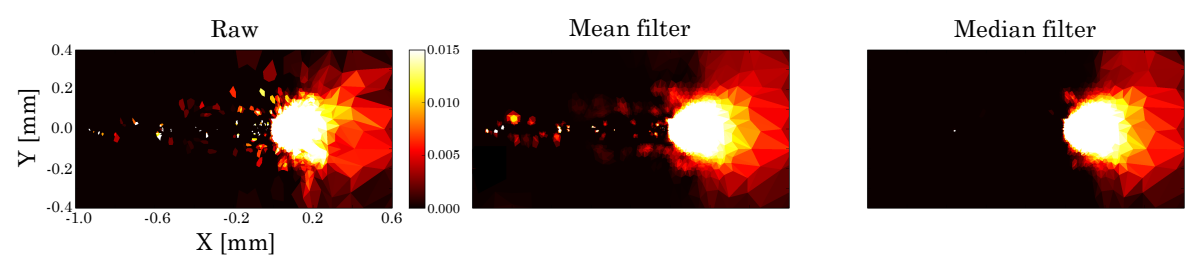

Figure 11: Equivalent plastic strains after unloading without a filter, with a mean filter and with a median filter having a size of 3 integration points.

Aberrant values are well reduced in the median regularization case while mean filter is not sufficient to obtain equivalent results. The impact of the median filter on the results is assessed via the plastic work, determined on the whole model and computed with Abaqus, during specific instants of the fatigue test (Fig. 미 (a)). No particular filtering impact is detected in terms of energies for a limited kernel dimension. Herein a size of three integration points is considered for the filter. Some tests were conducted with different regularization lengths and beyond three considered values, the relative error compared to raw data can reach 10\% (Fig. Ш (b)).

The ABAQUS command file is generated at each cycle and the regularization could be achieved conveniently at each one if necessary. Therefore, the median filter was chosen to reduce the noise and the results are filtered at each boundary conditions renewal. As the noise decreased and the methodology is perfectly monitored, a comparison can be carried out with the 'classical' pro- 


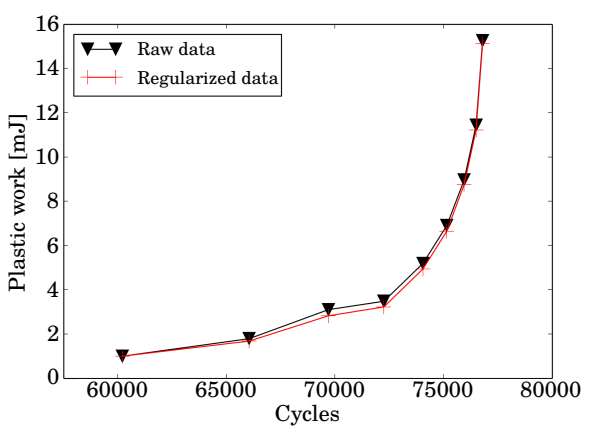

(a)

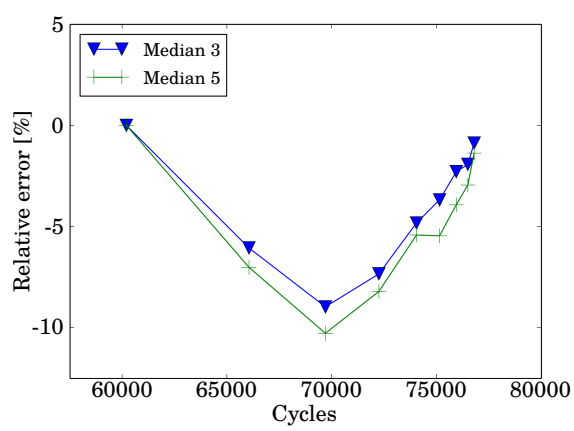

(b)

Figure 12: On the left, the plastic work over cycles for raw and filtered data (median filter with a size of 3 integration points) and on the right, relative errors compared to raw data for median regularization with 3 and 5 values.

jective approach.

\subsection{Comparison with the projective approach}

To validate the interest of DIC-FEM hybrid approach, the displacements residue was analyzed. As a reminder, the residue corresponds to the difference between experimental and analytical (in the projective method) or FEM displacement fields (in the hybrid approach case); FEM displacements fields are extracted from the ABAQUS result file. In the case of the Williams projective method (Section 2.3.2), the location of the highest residue (Fig. 四) coincided quite well with the Irwins model. This made it possible to highlight the limits of the Williams base, which did not consider a certain number of non-linearities, including plasticity.

The evolution during the fatigue test of the mean residues values, corresponding to the absolute value of differences between the measured displacement field and the extracted field (in the two main directions), are plotted in Figure [03. Before 65,000 cycles, the results obtained by projection and coupling are similar so that this latter brings no improvement. After 65000 cycles, the residue obtained with the hybrid method are consistently smaller than with the projective method. This limit is likely to correspond to the transition from small to large 
scale yielding conditions.
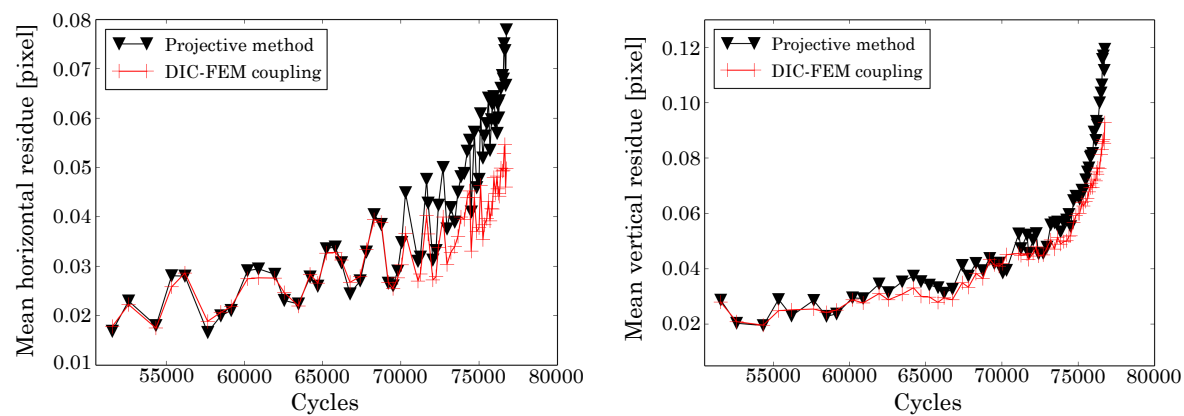

Figure 13: Evolution of absolute value of identification mean residues for projective and coupling methodologies in the horizontal (on the left) and the vertical (on the right) directions.

For example, Figure प4 shows vertical residue fields in projection and DICFEM coupling cases for a crack length of $9.5 \mathrm{~mm}$ (corresponding approximately to 76,000 cycles). As previously for the full-field projection (Fig. 四), an important localization is noticed at the crack tip, highlighting the assumed plasticity, whereas the coupling approach engenders a different residues distribution. Whether for projective correlation or DIC-FEM coupling, there is a significant localization of residues on the flanks of the crack that could be explained by contacts of the lips of the crack. Solanki [42] pointed out that contact consideration is essential to estimate crack opening. In addition to the effects of plasticity, graphite cast irons are known to exhibit crack closure effects due to material roughness [22] or detachment of graphite particles [13]. Furthermore, Camas et al. [9] showed the crack curvature can increase the plastic area in the case of 3D finite element computations. Thus, if the curvature is not taken into account, it is possible that the plastic zone and therefore the wake are underestimated. This may then contribute to the residue (in addition to contact) along the lips of the crack.

Nonetheless, a limitation can be specified, since the comparison is conducted on the 'classical' Williams' expansion series with orders from 1 to 7 . However, if a higher value of the maximum positive Williams' order is considered, the 

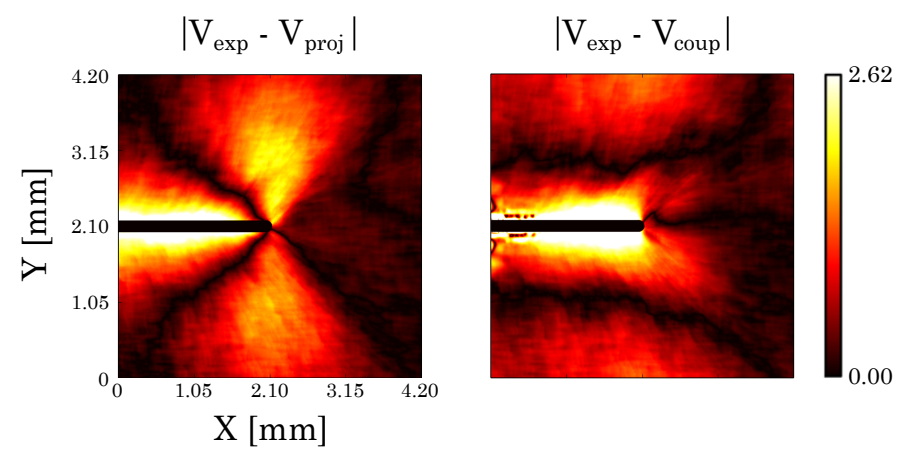

Figure 14: On the left, the vertical identification residues (in $\mu \mathrm{m}$ ) for the projective approach and on the right for the DIC-FEM coupling.

boundary conditions could be better described [IT]. Furthermore, if negative Williams' orders are taken into account, some local effects close to the crack tip could be considered [18]. To ensure the robustness of the projection, only the singular and subsingular terms are used.

As it can be observed in Figure [4, a part of the residues remains and could be attributed to the constitutive law; in the next part, this aspect will be investigated.

\subsection{Impact of the hardening law}

As FEM computations are driven by experimental displacements, one may wonder what is the impact of the material constitutive law on the results especially in terms of the plastic zone size. The elastic characteristics and the yield strength present a limited uncertainty unlike the strain hardening. To evaluate this aspect, computations with a linear kinematic hardening are compared with those assuming a perfectly plastic behavior. The plastic zone shapes are extracted from von Mises stress fields using the yield strength. Figure $\square .5$ shows the results at two values of the stress intensity factor range that correspond to the beginning and close to the end of the coupling approach (6 and $9 \mathrm{~mm}$ respectively).

The plastic zone sizes match well for the two investigated plastic laws. So 


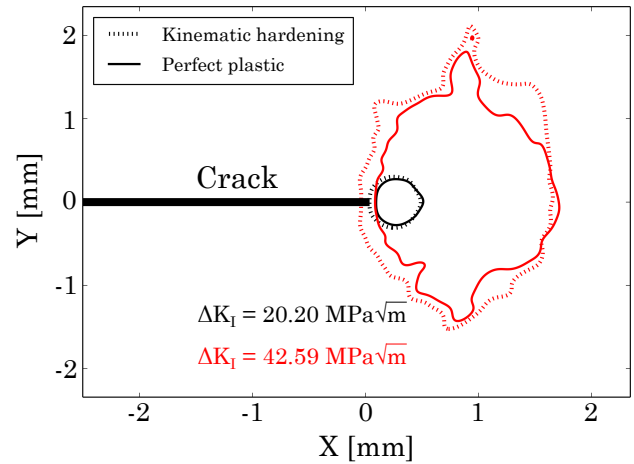

Figure 15: Plastic zone sizes close to the beginning and the end of the coupling computations for the Prager's linear kinematic hardening law and the perfectly plastic cases.

the impact in terms of plastic contours is limited when the hardening parameter is modified and the plastic shape must be driven mainly by the yield strength, in agreement with Irwin's model (Eq. 四). At this stage, interesting results were obtained using a rough estimate of material parameters. Nonetheless, a yield strength optimization could be achieved by an inverse identification method such as Finite Elements Method Updating (FEMU) [2]. For this latter, the framework is the same as for the coupling so that it could easily be added in the future.

Finally, only one supplementary parameter compared to Williams projective approach, i.e. the yield strength added in the finite elements model, improves the displacements description in a cracked medium (in relation to the first method).

\subsection{Plastic zone evolution and comparison with the Irwin's model}

As before, plastic contours are obtained via the von Mises criterion. In Figure ए6, plastic zones are drawn centred on the crack tip.

First, a large plastic zone size expansion is noticed when the number of cycles and crack length increase. Three cases are dealt with to observe the plasticity evolution from small to large scale yielding condition. The small scale yielding 

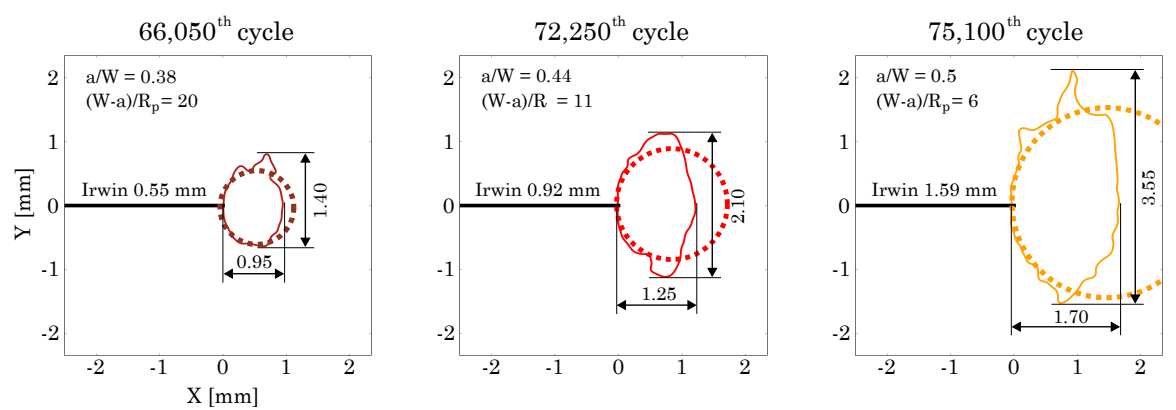

Figure 16: Plastic zone shapes identified from our hybrid methodology; the radius of Irwin's plastic zone, plotted as a dotted line circle, is given for comparison.

condition is stated via the ratio [2]]:

$$
\frac{W-a}{R_{p}}>25
$$

with $W$ the specimen width, $a$ the crack length and $R_{p}$ the Irwin's radius. When this ratio becomes less than 25 , small scale yielding can no longer be assumed. Herein, at the $66,050^{\text {th }}$ cycle, a ratio of 20 indicates the onset of large scale yielding while at the end of the test, i.e $75,100^{\text {th }}$ cycle, a ratio of 6 is reached. During the large scale yielding regime, plastic zone sizes increase rapidly from 1.4 to $3.5 \mathrm{~mm}$. This is a considerable evolution as only 9,000 cycles separate this two extreme cases. Despite the use of 7,500 finite elements, a mesh refinement could be necessary to improve plastic zone size definition especially for the last steps of the crack propagation.

The coupling results can be compared with those of Irwin which is defined for a perfect plastic behavior in a confined plasticity and plane stress cases [ $[8]$. DIC-FEM results present an interesting similarity with Irwin's model regardless of SIF level especially during large scale yielding conditions. Indeed, at the $66,050^{\text {th }}$ cycle, the shift between Irwin and DIC-FEM reaches $0.15 \mathrm{~mm}$ and it remains in the same order of magnitude with the crack propagation from 0.59 to $1.48 \mathrm{~mm}$ at respectively $72,250^{\text {th }}$ and $75,100^{\text {th }}$ cycles. With a finer mesh, the description of the plastic zone contour could improve especially for plastic zones 
larger than $1 \mathrm{~mm}$ in diameter.

Moreover, the similarity between Irwin's radius and DIC-FEM coupling validates the relevance of Irwin use for the mask definition in the projective approach. In this context, the impact of using a mask based on the evolving Irwin model, and no longer constant as carried out in Section 2.3, can be analyzed. Thus, the stress intensity factors obtained in this previous section by the projective approach are used to define the evolutionary Irwin mask. Figure $\square 0$ shows the evolution of the SIF during the fatigue test cycles for constant and evolving masks.

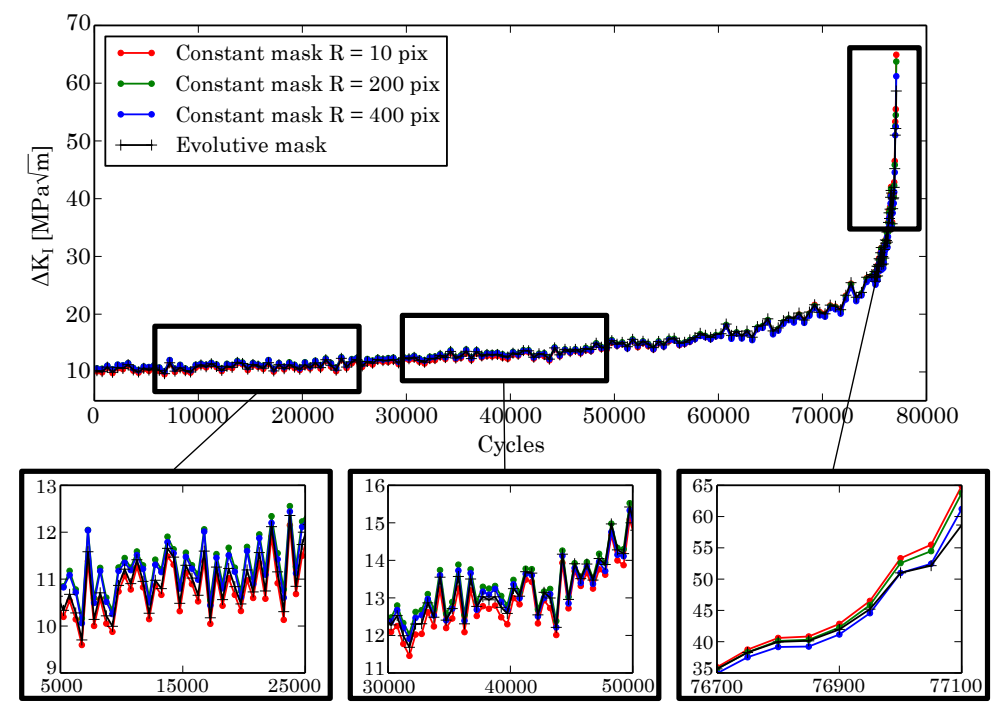

Figure 17: The stress intensity factor versus number of cycles for the constant and evolutive masks in projective DIC.

From the beginning of the test until 40,000 cycles, a limited difference of about $0.5 \mathrm{MPa} \sqrt{\mathrm{m}}$ has been observed between the evolutive and the larger constant masks. Although small, this difference is not negligible and could have an impact on the identification of Paris laws. In the last cycles of the crack growth test, the impact of plasticity on the evaluation of SIF increases and deviations of several $\mathrm{MPa} \sqrt{\mathrm{m}}$ are observed. Fig. $\square$ also shows that the curves obtained 
with constant masks constitute envelope curves for the one obtained with the evolutive mask during almost the whole test.

Therefore, the reliability of Irwin's model makes it possible to define a suitable mask for projective DIC. Then, using a mask as close as possible to the real plastic zone allows to measure more objective SIF values. However, for tests in high cycle fatigue, i.e. with a limited plastic zone size throughout most of the test, the impact of the mask remains limited as observed by Mokhtarishirazabad et al. [ZZ]. Fatigue crack growth tests closer to low cycle fatigue, i.e. with a larger plastic zone size as compared to the crack length, should be performed to fully appreciate the benefit of the DIC-FEM coupling methodology.

The DIC-FEM coupled approach does not take into account fatigue crack shape, material heterogeneities such as grains sizes and their orientation or graphite impacts, but on average, plastic behavior is well described. From our knowledge, it is the first time that some plastic contours, from an hybrid experimental-numerical approach, are compared with Irwins model. The results confirm the accuracy of the pioneering approach provided by Irwin in 1960 and cited in $[8]$.

\section{Conclusion}

For fatigue design, the plastic zone is often neglected although this latter plays a considerable role in the crack velocity. Therefore, having a reliable method to estimate the plasticity evolution at the crack tip would improve the design structures. In this study, an original hybrid formulation is presented, the procedure combines the accuracy and the large amount of information provided by Digital Image Correlation with the wide possibilities range of Finite Elements Method. The hybrid DIC-FEM coupling methodology relies on the application of measured displacements fields as boundary conditions into elastic-plastic computations, provided by DIC and FEM respectively. It enables to apply cycles 
between each new boundary conditions in order to be close to reality and to observe the plasticity evolution from small to large scale yielding conditions.

The developed approach was applied in the case of a standard fatigue crack propagation test, i.e. at constant maximum load. A median filter regularization is proposed to reduce the noise in the experimental data. It was shown that this regularization brings no important modification of the results especially in terms of plastic work. Then, the residues between measured displacements and FEM results are lower than those obtained between measured displacements and Williams' basis projective approach (with a classical order range from 1 to 7 as presented in [20]]). The residues decrease validates the added value of the DIC-FEM methodology. Furthermore, the approach provides the stress and strain fields at the crack tip that allow to evaluate the shape and extent of the plastic zone over cycles. Some computations were conducted considering either a linear kinematic hardening or a perfectly plastic behavior but no significant difference was noticed in terms of plastic zone size. DIC-FEM results are close to those of Irwin's plastic zone size although this latter seems to be conservative. To the author's knowledge, this is the first time such results are obtained from experiments thanks to the hybrid method. Thus, they validate the use of the Irwin's plastic radius for reliable mask definition in the DIC projective approach.

Nonetheless, the DIC-FEM coupling approach presents some restrictions. The main hypothesis of this study is that we only consider a perfectly straight propagation with no bifurcations in a two-dimensional medium. However, microtomographic measurements on some cracked specimens have shown the presence of curved cracks. A difference in crack advance between the core and the surface of about $1 \mathrm{~mm}$ was observed. To go further, the use of X-ray micro-tomography seems inevitable. Moreover, the crack lips contacts are not taken into account despite the significant impact of roughness on the crack closure especially for ductile cast irons [I7]]. Nonetheless, the loading ratio is kept positive, herein the crack closure effects must be limited. 
Finally, some advantages and prospects for the coupling methodology can be cited such as:

1. A suitable law for the contact between the crack faces in mixed mode conditions could be the subject of future investigations in order to deal with negative load ratio.

2. The coupling approach is adaptive and for example high temperature cases could be treated by modifying the material constitutive law. In this case, plasticity is more important. The procedure could be particularly adapted to this case of generalized plasticity.

3. Complex loadings, e.g. random with several overloads, could be dealt with to appreciate the influence on crack velocity.

4. In order to consider the crack shape and to get free from the plane stress/strain hypothesis, the achievement of an in situ fatigue test with micro-tomography could help to go further. Indeed, an extension of the DIC-FEM methodology is practicable because digital image correlation in volumes is possible with a ductile cast iron [24]. The graphite nodules and the ferritic matrix present a good contrast in X-ray micro-tomography [2.9] allowing to have a good 3D speckle pattern. Then, such Digital Volume Correlation could be coupled with 3D FEM computations [35].

\section{Acknowledgements}

The authors wish to thank the generous support of PSA Group for this survey.

\section{References}

[1] J Abanto-Bueno and J Lambros. Investigation of crack growth in functionally graded materials using digital image correlation. Engineering Fracture Mechanics, 69, 2002. 
[2] S Avril, M Bonnet, AS Bretelle, M Grediac, F Hild, P Ienny, F Latourte, D Lemosse, S Pagano, E Pagnacco, et al. Overview of identification methods of mechanical parameters based on full-field measurements. Experimental Mechanics, 48(4):381, 2008.

[3] B Bay, T Smith, D Fyhrie, and M Saad. Digital volume correlation: threedimensional strain mapping using x-ray tomography. Experimental mechanics, 39(3):217-226, 1999.

[4] T Belytschko and T Black. Elastic crack growth in finite elements with minimal remeshing. International journal for numerical methods in engineering, 45(5):601-620, 1999.

[5] T Belytschko and H Chen. Singular enrichment finite element method for elastodynamic crack propagation. International Journal of Computational Methods, 1(01):1-15, 2004.

[6] G Berkooz, P Holmes, and JL Lumley. The proper orthogonal decomposition in the analysis of turbulent flows. Annual review of fluid mechanics, 25(1):539-575, 1993.

[7] G Besnard, F Hild, and S Roux. Finite-element displacement fields analysis from digital images: application to portevin-le châtelier bands. Experimental Mechanics, 46(6):789-803, 2006.

[8] D Broek. Elementary engineering fracture mechanics. Springer Science \& Business Media, 2012.

[9] D Camas, J Garcia-Manrique, and A Gonzalez-Herrera. Crack front curvature: Influence and effects on the crack tip fields in bi-dimensional specimens. International Journal of Fatigue, 44:41-50, 2012.

[10] E Charkaluk, R Seghir, L Bodelot, JF Witz, and P Dufrénoy. Microplasticity in polycrystals: a thermomechanical experimental perspective. $E x$ perimental Mechanics, 55(4):741-752, 2015. 
[11] N Dahdah, N Limodin, A El Bartali, JF Witz, R Seghir, E Charkaluk, and JY Buffiere. Damage investigation in a319 aluminium alloy by x-ray tomography and digital volume correlation during in situ high-temperature fatigue tests. Strain, 52(4):324-335, 2016.

[12] PY Decreuse, S Pommier, M Poncelet, and B Raka. A novel approach to model mixed mode plasticity at crack tip and crack growth. experimental validations using velocity fields from digital image correlation. International Journal of Fatigue, 42:271-283, 2012.

[13] P Dierickx. Etude de la microstructure et des mécanismes d'endommagement de fontes $G$. S. ductiles. Influence des traitements thermiques de ferritisation. $\mathrm{PhD}$ thesis, 1996.

[14] J Dolbow, N Moës, and T Belytschko. An extended finite element method for modeling crack growth with frictional contact. Computer methods in applied Mechanics and engineering, 190(51-52):6825-6846, 2001.

[15] T Elguedj, A Gravouil, and A Combescure. A mixed augmented lagrangianextended finite element method for modelling elastic-plastic fatigue crack growth with unilateral contact. International Journal for Numerical Methods in Engineering, 71(13):1569-1597, 2007.

[16] RP Gangloff, DC Slavik, RS Piascik, and RH Van Stone. Small-Crack Test Methods, ASTM STP 1149, chapter Direct Current Electrical Potential Measurement of the Growth of Small Cracks. West Conshohocken (PA, USA), 1992.

[17] G Greno, J Otegui, and R Boeri. Mechanisms of fatigue crack growth in austempered ductile iron. International Journal of Fatigue, 22:35-43, 1999.

[18] R Hamam, F Hild, and S Roux. Stress intensity factor gauging by digital image correlation : application in cyclic fatigue. Strain, 43, 2007. 
[19] C Henninger, S Roux, and F Hild. Enriched kinematic fields of cracked structures. International Journal of Solids and Structures, 47(24):3305$3316,2010$.

[20] J Hosdez, JF Witz, C Martel, N Limodin, D Najjar, E Charkaluk, P Osmond, and F Szmytka. Fatigue crack growth law identification by digital image correlation and electrical potential method for ductile cast iron. Engineering Fracture Mechanics, 182:577-594, 2017.

[21] Astm International. Standard test method for plane-strain fracture toughness of metallic materials. ASTM 399-90, 2007.

[22] MN James and L Wenfong. Fatigue crack growth in austempered ductile and grey cast irons-stress ratio effects in air and mine water. Materials Science and Engineering: A, 265(1-2):129-139, 1999.

[23] J Lachambre. Développement d'une méthode de caractérisation 3D des fissures de fatigue à l'aide de la corrélation d'images numériques obtenues par tomographie X. PhD thesis, INSA de Lyon, 2014.

[24] N Limodin, J Réthoré, JY Buffière, A Gravouil, F Hild, and S Roux. Crack closure and stress intensity factor measurements in nodular graphite cast iron using three-dimensional correlation of laboratory x-ray microtomography images. Acta materialia, 57(14):4090-4101, 2009.

[25] P Lopez-Crespo, A Shterenlikht, JR Yates, EA Patterson, and PJ Withers. Some experimental observations on crack closure and crack-tip plasticity. Fatigue \& Fracture of Engineering Materials \& Structures, 32(5):418-429, 2009.

[26] N Moës, J Dolbow, and T Belytschko. A finite element method for crack growth without remeshing. International journal for numerical methods in engineering, 46(1):131-150, 1999.

[27] N Moës, A Gravouil, and T Belytschko. Non-planar 3d crack growth by the extended finite element and level sets-part i: Mechanical model. Inter- 
national Journal for Numerical Methods in Engineering, 53(11):2549-2568, 2002.

[28] M Mokhtarishirazabad, P Lopez-Crespo, B Moreno, A Lopez-Moreno, and M Zanganeh. Evaluation of crack-tip fields from dic data: a parametric study. International Journal of Fatigue, 89:11-19, 2016.

[29] K Mukherjee, S Fæster, N Hansen, AB Dahl, C Gundlach, JO Frandsen, and A Sturlason. Graphite nodules in fatigue-tested cast iron characterized in 2d and 3d. Materials Characterization, 129:169-178, 2017.

[30] T Ogawa and H Kobayashi. Near threshold fatigue crack growth and crack closure in a nodular cast iron. Fatigue $\mathcal{E}$ Fracture of Engineering Materials ES Structures, 10(4):273-280, 1987.

[31] JJ Orteu. 3-d computer vision in experimental mechanics. Optics and Lasers in Engineering, 47(3-4):282-291, 2009.

[32] PC Paris and F Erdogan. A critical analysis of crack propagation laws. Journal of basic engineering, 85(4):528-533, 1963.

[33] S Pommier and P Bompard. Bauschinger effect of alloys and plasticityinduced crack closure: a finite element analysis. Fatigue and Fracture of Engineering Materials and Structures, 23(2):129-140, 2000.

[34] W Prager and PG Hodge. Theory of perfectly plastic solids. 1951.

[35] H Proudhon, J Li, F Wang, A Roos, V Chiaruttini, and S Forest. 3d simulation of short fatigue crack propagation by finite element crystal plasticity and remeshing. International Journal of Fatigue, 82:238-246, 2016.

[36] P Rabbe, HP Lieurade, and A Galtier. Essais de fatigue. partie i. Techniques de l'ingénieur. Matériaux métalliques, (M4170):M4170-1, 2000.

[37] J Réthoré, A Gravouil, and A Combescure. An energy-conserving scheme for dynamic crack growth using the extended finite element method. International Journal for Numerical Methods in Engineering, 63(5):631-659, 
2005.

[38] J Réthoré, F Hild, and S Roux. Extended digital image correlation with crack shape optimization. International Journal for Numerical Methods in Engineering, 73(2):248-272, 2008.

[39] C Roux-Langlois, A Gravouil, MC Baietto, J Rethore, F Mathieu, F Hild, and S Roux. Dic identification and x-fem simulation of fatigue crack growth based on the williams' series. International Journal of Solids and Structures, 53:38-47, 2015.

[40] R Seghir, JF Witz, and S Courdert. Yadics digital image correlation 2/3D software. http://yadics.univ-lille1.fr/.

[41] M Shakoor, A Buljac, J Neggers, F Hild, TF Morgeneyer, L Helfen, M Bernacki, and PO Bouchard. On the choice of boundary conditions for micromechanical simulations based on 3d imaging. International Journal of Solids and Structures, 112:83-96, 2017.

[42] K Solanki, SR Daniewicz, and JC Newman Jr. Finite element analysis of plasticity-induced fatigue crack closure: an overview. Engineering Fracture Mechanics, 71(2):149-171, 2004.

[43] M Sutton, S McNeill, J Helm, and Y Chao. Advances in two-dimensional and three-dimensional computer vision. In Photomechanics, pages 323-372. Springer, 2000.

[44] JM Vasco-Olmo, FA Díaz, A García-Collado, and R Dorado-Vicente. Experimental evaluation of crack shielding during fatigue crack growth using digital image correlation. Fatigue \& Fracture of Engineering Materials 8 Structures, 38(2):223-237, 2015.

[45] JM Vasco-Olmo, MN James, CJ Christopher, EA Patterson, and FA Díaz. Assessment of crack tip plastic zone size and shape and its influence on crack tip shielding. Fatigue $\&$ Fracture of Engineering Materials $\&$ Structures, 39(8):969-981, 2016. 
[46] M Williams. On the stress distribution at the base of a stationary crack. ASME J. Appl. Mech., 24, 1957. 